\title{
Ground Autonomy for an Aging Spacecraft
}

\author{
Charles J. LaBonde*, Sierra Flynn ${ }^{\dagger}$, Matt Muszynski ${ }^{\ddagger}$, Sean Ryan $^{\S}$, Deb McCabe ${ }^{\mathbb{\Phi}}$, and Emily Pilinskill \\ University of Colorado - Laboratory for Atmospheric and Space Physics (LASP), Boulder, CO, 80303, United States
}

As it approaches the sixteenth of a 5 year prime mission, NASA's Solar Radiation and Climate Experiment (SORCE) mission continues to meet and exceed all science requirements while operating with severely degraded batteries. By 2013, the batteries had degraded such that the On Board Computer $(\mathrm{OBC})$ could not be powered through eclipse. This prevents science data collection in eclipse and erases over $99 \%$ of all stored science and engineering telemetry. To mitigate these problems, the Flight Operations Team (FOT) at the Laboratory for Atmospheric and Space Physics (LASP) adopted a new operations scheme. "Daylight Only Operations" (DO-OP) transitions the spacecraft from safemode to science mode every orbit. This involved heavily automating the transition process using ground autonomy to improve spacecraft recovery time to 6 minutes - down from 3 orbits of manual commanding. While highly successful, this method of operations poses daily challenges that must be overcome using increasingly complex ground software.

\footnotetext{
*Command Controller - Graduate Student Lead

Command Controller - Graduate Student Lead

¥Command Controller - Graduate Student Lead

$\S$ Mission Operations Manager

"Flight Director

"Flight Controller
} 


\section{Introduction}

SORCE launched on January 25, 2003 on a Pegasus XL rocket into a $645 \mathrm{~km}, 40^{\circ}$ inclination LEO orbit. The mission provides precise measurements of solar radiation for NASA's Earth Science Enterprise with 4 instruments: the Spectral Irradiance Monitor (SIM), the Solar Stellar Irradiance Comparison Experiment (SOLSTICE), the Total Irradiance Monitor (TIM), and the XUV Photometer System (XPS). Over the past 15 years SORCE made significant contributions to the sun-climate records of the Total Solar Irradiance (TSI) and Spectral Solar Irradiance (SSI). SORCE also provided cross calibration with several successor missions including the Total Solar Irradiance Transfer Experiment (TCTE) and the Total and Spectral Irradiance Sensor (TSIS) launched in 2013 and 2017 respectively.

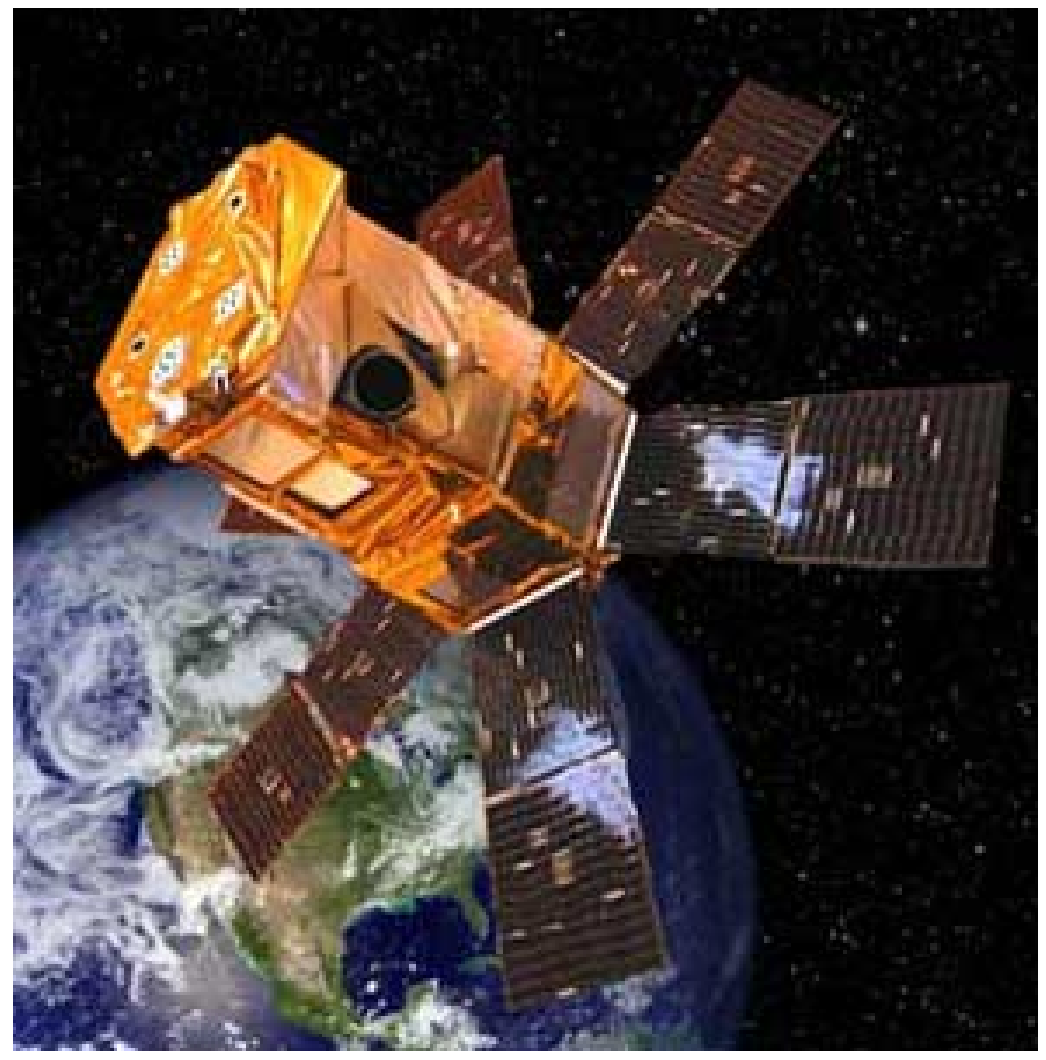

Fig. 1 SORCE spacecraft

SORCE uses a nickel-hydrogen battery with 11 Common Pressure Vessels (CPV). Each CPV contains 2 electrochemical cells and was originally designed for a 6 year lifespan. In 2011, after 8 years in operation, the spacecraft experienced 2 battery cell failures, which were followed by 3 more in 2012 and 2 in 2013 . As the failures continued, it became clear that the spacecraft could no longer power all loads throughout the orbit and in 2011, the FOT began turning off some instruments in eclipse to save power. By the fifth failure of 2012, minimum voltages had dropped to the point that no science instruments could be left on while not in sunlight. The FOT used Relative Time Sequences (RTS) to perform science operations in day lit portions of the orbit and turn off all instruments during eclipse. After the 2013 cell failures the battery could no longer power the OBC through eclipse. Without its main computer, SORCE could no longer use the Star Tracker and Fine Sun Sensor (FSS) in eclipse. This resulted in backflips at sunrise, a behavior that occurs when natural perturbations on the spacecraft's attitude during eclipse cause the solar array to point $180^{\circ}$ from the sun at eclipse exit. The Attitude Control System (ACS) maneuvers the spacecraft back to the sun, but this slow maneuver is hazardous to the spacecraft's health as the battery continues discharging, effectively extending eclipse duration up to 20 minutes.

To prevent backflips, the FOT implemented a $0.5 \mathrm{Nms}$ momentum bias around the instrument boresight (which points normal to the solar panels) during orbit day. In eclipse, the momentum bias spins the spacecraft as the RWAs spin down from friction, allowing it to remain gyroscopically stable throughout eclipse. This prevents SORCE from experiencing backflips upon exiting eclipse. The momentum bias saves significant power as it allows the main ACS 
components (Reaction Wheel Assemblies (RWA), Magnetic Torquer Bars (MTB), Three Axis Magnetometer (TAM), and the Star Tracker (ST)) to be off in eclipse without sacrificing pointing performance.

Without being able to power the OBC in eclipse, the FOT developed a new concept of operations that allowed the spacecraft to be powered off before eclipse entrance and power on at eclipse exit, called DO-OP mode. This method of operations allowed the spacecraft to continue to perform science and reduce the risk of another battery failure.

\section{Daylight Only Operations}

In 2014, SORCE implemented DO-OP. This method of operations powers down all loads in eclipse except for the Attitude and Power Electronics (APE) backup computer. The APE is the reptilian brain of the spacecraft and can only control the most basic functions such as pointing the spacecraft coarsely at the sun and regulating the power from the solar arrays. It is the last major component that can remain powered before the batteries are depleted. Because of momentum biasing, SORCE's solar panels nominally point within a few degrees of the sun at sunrise. The solar arrays detect the sun and turn on the ACS components to despin the spacecraft.

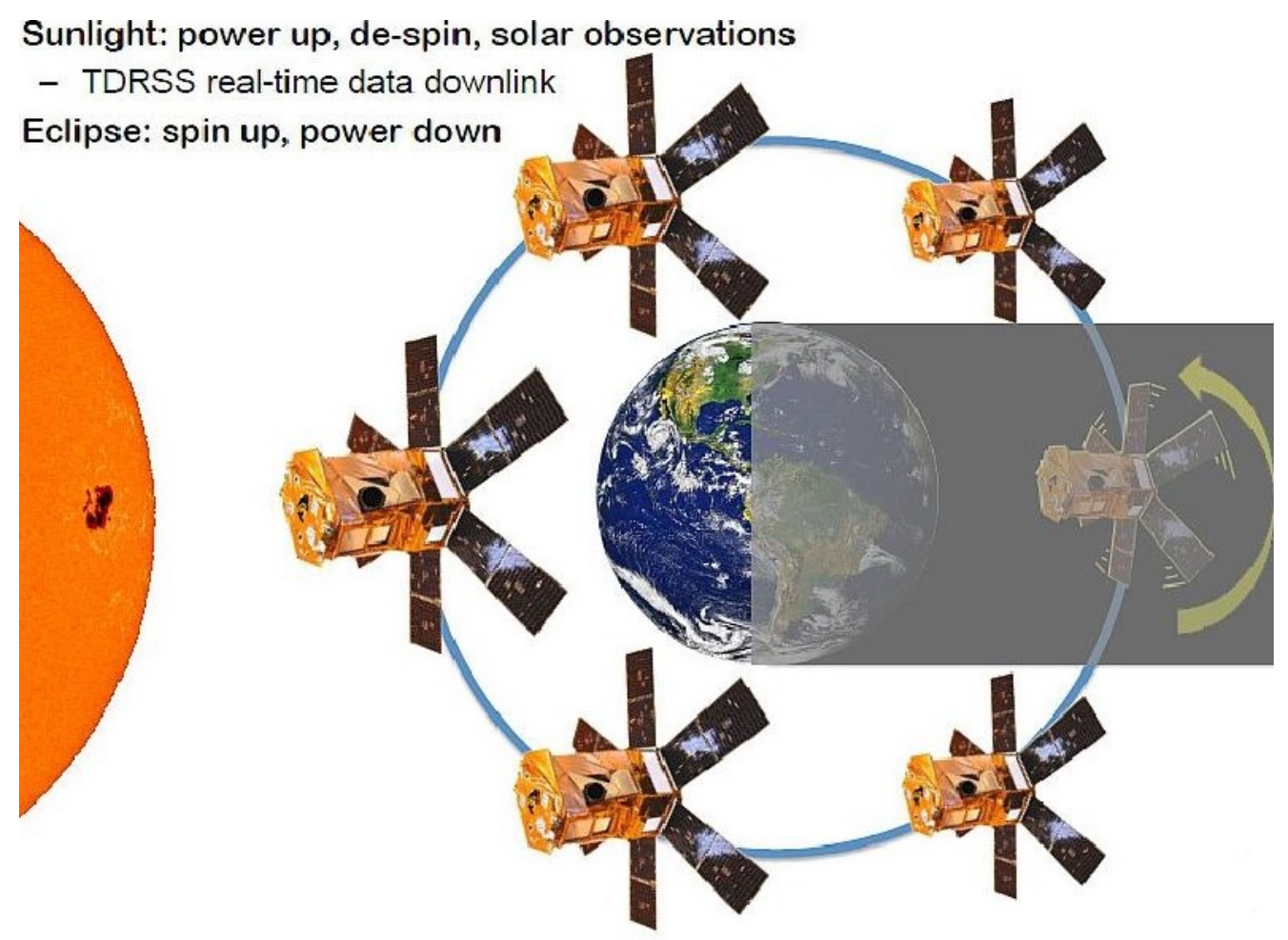

Fig. 2 DO-OP CONOPS

During this time, the OBC is turned on and a nominal eclipse exit RTS configures the spacecraft. This includes turning on all the science instruments, transferring control of the spacecraft from the APE to the OBC, and exiting safemode. The spacecraft is now in contingency mode where no science is taken, but it is in a stable state awaiting further instructions. Once the ground automation verifies the spacecraft state of health and takes corrective action if necessary, an Absolute Time Sequence (ATS) is loaded to the spacecraft. Contained in the ATS are commands to transition from contingency mode to normal mode, conduct science, and run a safemode RTS to safely configure for eclipse at the end of the sun lit period. If the spacecraft does not configure itself for eclipse nominally via ATS, ground autonomy attempts to execute the safemode sequence. If all else fails, the spacecraft executes a contingency sequence when the solar array current decreases below a threshold value, signaling eclipse entrance.

Since almost all science and engineering data is erased during eclipse when the OBC turns off, the FOT relies on the Space Network's (SN) Tracking and Data Relay Satellite (TDRS) contacts to monitor the spacecraft and collect science data. Ground contacts are used when orbital geometry permits. Nominally, SORCE will have a 10 minute TDRS contact 1 minute after sunrise. This "safety contact" allows ground automation to verify the state of health and configure the 
spacecraft. The 1 minute delay after sunrise allows the spacecraft to boot to a default configuration. After the safety contact, SORCE requires a 25 minute TDRS contact. This occurs while SORCE conducts science experiments and allows the data to be dumped to the real time stream during the TDRS contact. When ground stations are available, they will be scheduled at the end of orbit day. Since the data rate for ground contacts is significantly higher than TDRS contacts, all science and engineering data can be down linked before the OBC is turned off and the spacecraft enters eclipse.

\section{A. DO-OP Operation Modes}

In DO-OP, SORCE progresses through several different modes of operation. They include safemode, contingency mode, and normal/science mode. Encompassing these modes is a broader operations concept called brownout mode. In November 2016, despite turning off the OBC and instrument suite, another battery cell failure reduced the overall charge capacity below the threshold required to power the APE in eclipse. This causes the APE to "brown out" and turn off. At sunrise, the APE boots to an unalterable launch version of flight software. This default Flight Software (FSW) does not account for component degradation and configures the spacecraft to an undesirable state. The default APE FSW will not turn the OBC on. Ground autonomy will automatically turn the OBC on, starting an onboard process that switches the APE to the updated version of FSW and prepares the rest of the spacecraft for nominal operations.

At sunrise, in both brownout and non-brownout operations, the spacecraft will default to safemode. Safemode is the survival state of the spacecraft and includes only basic operations. In this state, the APE is in control of the spacecraft. The APE can't use information from the star tracker or FSS and can't conduct science. It is normally used when the spacecraft experiences a significant anomaly such as a component failure.

Once SORCE is correctly configured, the spacecraft will automatically exit safemode and enter contingency mode. In this mode, the $\mathrm{OBC}$ is in control of the spacecraft. No science can be conducted in this mode. Contingency mode is similar to safemode, but has increased functionality. This state is used when SORCE is functioning correctly, but not conducting science. In some cases, SORCE will regress from science mode to contingency mode when science cannot be conducted.

From contingency mode, the ATS will command the spacecraft to enter normal/science mode. In this mode, SORCE uses the star tracker and FSS to point precisely at the Sun and conduct science experiments. Originally, SORCE operates in this mode unless an anomaly occurs and the Fault Detection and Correction (FDC) system commands the spacecraft to contingency mode or safemode.

\section{Ground Autonomy}

There are several different elements of SORCE's ground autonomy that must work together to ensure the health and performance of the spacecraft. While the subsections below refer specifically to procedures written in the Colorado System Test Operational Language (CSTOL), these procedures have several layers of software to support them. At the core is the Operations and Science Instrument Support Command Control (OASIS-CC) software. This program allows the Mission Operations Center (MOC) to send commands to the spacecraft and capture real-time telemetry data. The front end for OASIS-CC, OASIS Interface Software (OIS), handles the ground communications with the stations. While OASIS-CC is capable of running real time telemetry checkers written in CSTOL, it can't process all data streams. Telemetry Data Processing (TDP) software is used that processes all raw forms of telemetry including real time data, recorded engineering housekeeping data, and recorded science data that is played back during a contact. Once processed, the Telemetry Quality and Status Monitor (TQSM) software can perform basic limit checking of the engineering data and alerts the FOT of limit violations or problems processing the data via a paging system.

This software allows the FOT to manually operate the spacecraft, but requires several CSTOL procedures to effectively automate the operations of the spacecraft with minimal human involvement. The procedures run from a command line within OASIS-CC. Up to 9 procedures may be run simultaneously. Each of these Control Language Processors (CLP) can be individually manipulated by designating a number, 1-8, before the command. Additionally, there is a User CLP that does not require a number by default. The User CLP is reserved for real time commanding whereas CLPs 1-8 are reserved for automation. Fig. 3 shows which procedures are running in each CLP and the procedures they start. The CLP numbers are inherited from previous missions and are trivial. The diagram includes 2 nd level sub-procedures such as comm setup and auto wd (the watchdog) that are referred to in later sections, but are not the focus of the paper and provided for reference. 


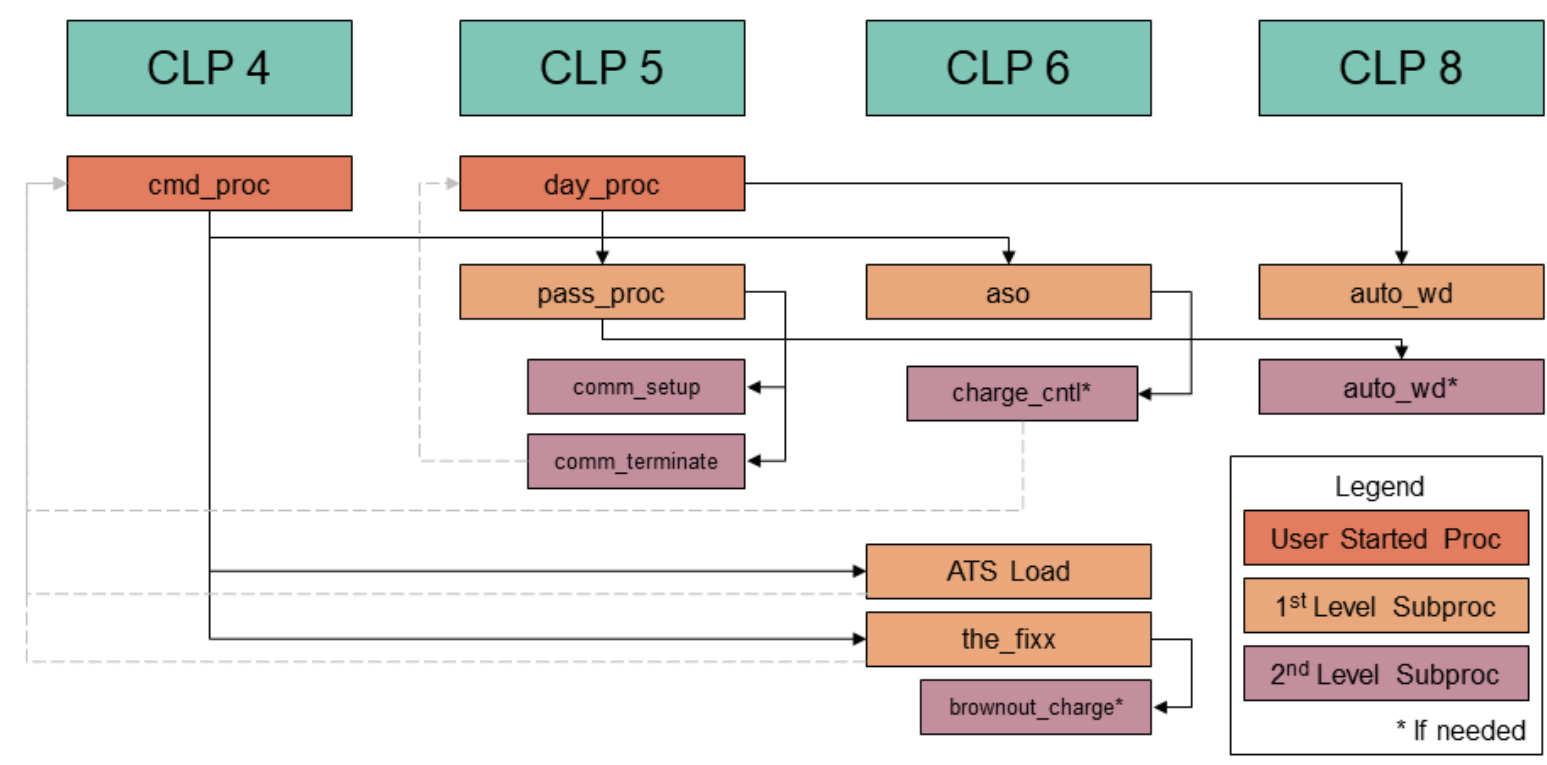

Fig. 3 SORCE CLP diagram

Most of the automation can be grouped into two types of procedures. The first group consists of the Day Procedure (day proc) and the Command Procedure (cmd proc). These scripts contain information and instructions for each orbit and contact in the current schedule. Day procs are used across all LASP missions to configure OASIS-CC and OIS to connect to either a ground station or TDRS terminal just before a planned contact. This procedure is also responsible for terminating connections at the end of the contact. The cmd proc contains the bulk of the unique automation required to operate SORCE in DO-OP mode. Both the day proc and the $\mathrm{cmd}$ proc are used to start other generic procedures at appropriate times relative to sunrise and sunset to transition SORCE safely to science mode and back to safe mode at the end of each orbit. Each of these procedures is updated daily during the week to account for schedule changes and planning time ranges.

The second group of procedures consists of the Pass Procedure (pass proc), Auto SORCE Orbit (ASO), the ATS Load Procedure (load proc), and The Fixx. These procedures, with the exception of the orbit specific ATS load, are generic procedures started by the day proc and cmd proc. They contain all the checks and commands required to autonomously configure the spacecraft from safemode to science mode over the course of an orbit. They also contain logic to reconfigure the spacecraft in the event of an anomaly. Only the load procs are generated on a regular basis to include new ATSs. The other procedures are updated as operational changes are required.

The final procedure, the watchdog, does not fit into either category, but is critical to the automation. The watchdog procedure periodically checks the CLPs to verify that each procedure is operating correctly. If it detects an error, the procedure uses TQSM to page the Flight Controller (FC) on call to alert them of the problem. To ensure that the watchdog is running, the day proc first starts the watchdog before moving on to configuring OASIS-CC for a contact.

To clarify which procedures are running at what time during the orbit, Fig. 4 depicts the general duration and occurrence of each procedure throughout orbit day. 


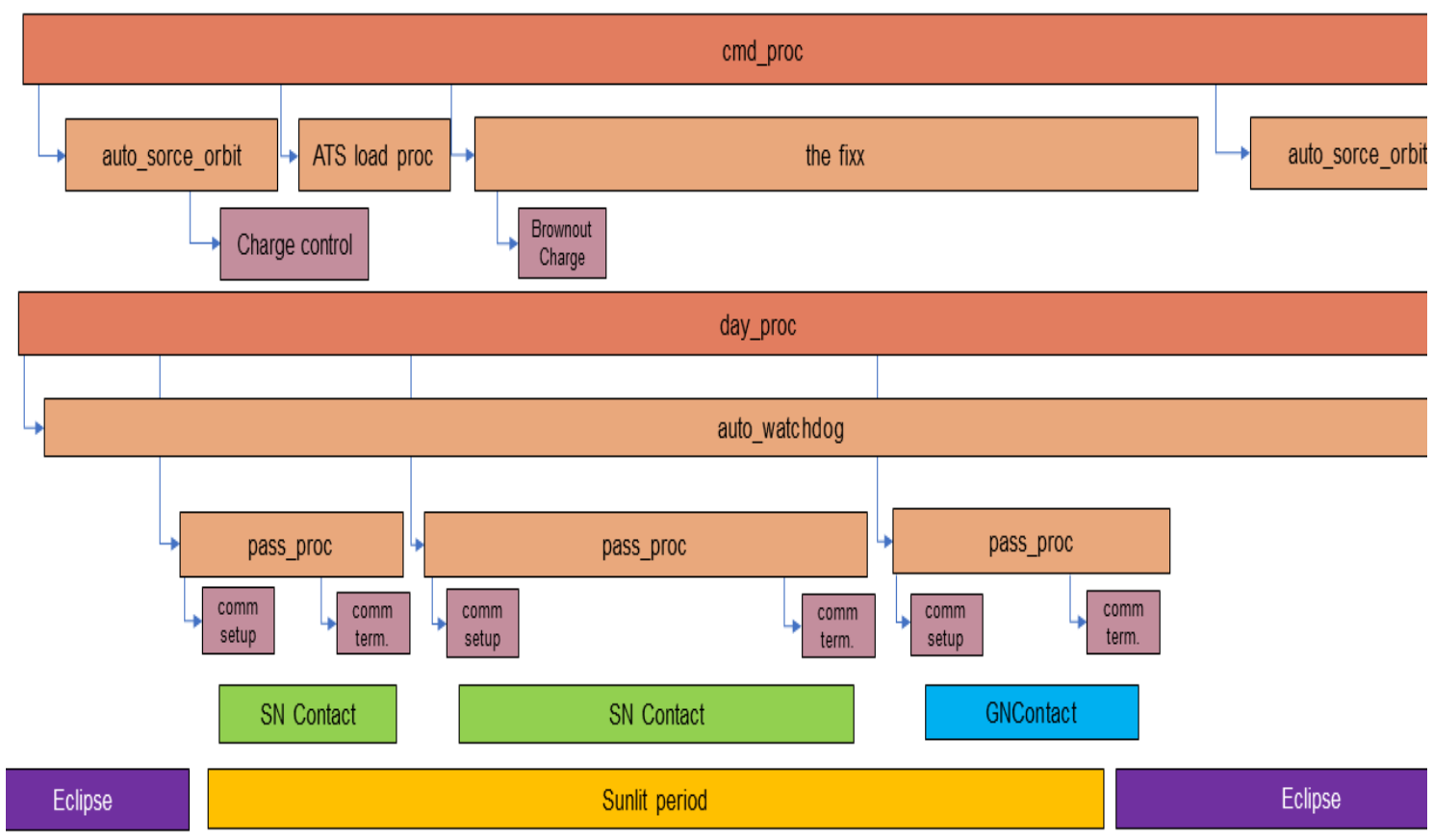

Fig. 4 SORCE ground autonomy over an orbit

Fig. 4 assumes that the day proc and cmd proc are started manually in eclipse. The sub procedures started by these procedures can run concurrently because they are in different CLPs, as described in Fig. 3. The cmd proc will start ASO, the ATS load proc, and the fixx during orbit day. After the fixx has completed, the cmd proc will automatically start ASO in preparation for the next orbit. The day proc will start the watchdog (auto watchdog) and pass proc. Pass proc will run during every contact and use the sub procedures comm (communication) setup and comm terminate at the beginning and end of each contact, respectively. At the bottom of Fig. 4 are the 10 minute TDRS safety contact, the 25 minute TDRS science contact, and the occasional ground contact at the end of orbit day.

The day proc, cmd proc, and ATSs are planned and created by a team of students that are also in charge of scheduling TDRS contacts. The students are known as Command Controllers (CC) and assist the FC in real time operations, sending commands to the spacecraft under the direction of the FC. The CCs are responsible for starting the procedures when SORCE is in eclipse. The FC is not involved in this process unless there are special circumstances or a problem has occurred. The CCs undergo rigorous training and must pass several certifications before they are allowed to plan, create, and start these procedures.

Altogether, these procedures allow SORCE to operate with minimal human intervention in a mode that would otherwise require 24/7 staffing and a large amount of manual commanding that introduces the opportunity for mistakes.

\section{A. Day Procedure}

The day proc is generated every day to ensure the most recent schedule changes in ground or TDRS contacts are incorporated. Every contact must be in this procedure since SORCE has no onboard knowledge of its contact schedule. By default, the transmitter is turned on for the duration of orbit day configured for TDRS contacts. For a ground contact, the onboard FSW monitors the AGC level of the carrier signal from a ground station. Once the AGC is consistently above a threshold for a predetermined amount of time, SORCE automatically configures for a high rate recorder dump to a ground station. The day proc's internal logic determines if it is running in the expected CLP and starts the watchdog when the procedure is initialized. If the day proc is started incorrectly by the $\mathrm{CC}$, the $\mathrm{FC}$ on duty receives a page to correct it. The procedure nominally contains the entire active schedule, which can range up to 2 weeks, even though it is updated 5 times a week. This is done to mitigate unforeseen problems with planning software, network issues, TDRS anomalies, and other circumstances that prevent the FOT from creating procedures. Near the end of the schedule, 
the day proc commands TQSM to page the FC, informing them that the day proc is near its end. Normally the FOT manually terminates the day proc and replaces it with a newer version each business day, but in the event of a scheduling error, this check alerts the FOT to create a new procedure with more contacts. The day proc's main function is starting the pass proc, which runs through the duration of each contact. The day proc passes the specific start and stop times, mask times, the station ID, eclipse entry time, and the expected data rate configuration to the pass proc before the contact is expected to start. If the day proc includes contacts that have already occurred, the day proc skips the old contacts before waiting for the next contact to start based on start time.

\section{B. Pass Proc}

The pass proc is a generic procedure that runs at specific times given by the day proc. In addition to setting up station connects via OIS, the pass proc allows the FOT to consistently monitor telemetry on each contact without significantly customizing scripts to run for different types of contacts. This procedure is the first line of defense in detecting and correcting anomalies. The beginning of the procedure checks that autonomy is correctly executing and restarts the watchdog if halted. It pre-populates several important telemetry points in OASIS-CC with unrealistic values. This ensures that the telemetry checks are not spoofed by stale data from the previous contact. At least three minutes before the contact starts, the procedure connects to the correct ground station or TDRS, and enables the telemetry flow and command ability. If the procedure is unable to connect to the specified terminal within 1 minute, an email is sent to inform the FC. The script attempts to connect to the backup systems in the case of a connection problem. If that fails, another email is sent to the FC and the pass proc tries to connect to the primary terminals one more time. If connections are still unsuccessful, the script sends a final email informing the FC.

If connections are successful, the script continues configuring OASIS-CC. On TDRS contacts the MOC can automatically send Ground Control Message Requests (GCMRs) through NASA's Space Network Access System (SNAS) software if certain conditions are met. This may include requesting forward link resweeps if no signal is detected from the SORCE.

After configuring OASIS-CC, the pass proc performs an initial telemetry check to make sure the OBC is turned on by the APE. If connections to the ground terminal are good but there is no data presence, a command is sent to turn on the OBC and jam the Uplink Card time to the current time. This is critical during brownout operations as the APE does not automatically turn on the OBC as the APE nominally boots to a default version of FSW. The script performs this action 4 times before requesting a forward link resweep to establish contact and repeat the process until telemetry indicates that the $\mathrm{OBC}$ is on.

Once the OBC turns on either from the APE or by ground command, pass proc performs a rigorous telemetry check. If at any point during this check telemetry indicates that the $\mathrm{OBC}$ is powered off, the script immediately turns it back on unless SORCE has entered eclipse. While TDRS contacts run up to 1 minute into eclipse to ensure all data is captured, ground station contacts can run further into eclipse. This catch prevents automation from trying to turn on the OBC after it nominally powers down at eclipse entrance. This saves power and reduces the chance that SORCE needlessly browns out in eclipse whatsoever.

At the beginning of the orbit, however, there is a chance that natural attitude perturbations such as nutation cause SORCE to exit eclipse pointed away from the sun. This is not ideal since SORCE will not have enough power to efficiently boot and return to science. If the spacecraft is off pointed more than $45^{\circ} 5$ minutes after eclipse exit, the script pages the FC and informs them that SORCE is not solar pointed. This alarm is likely indicative that SORCE did not achieve the correct configuration before entering eclipse on the previous orbit and triggers the FOT to investigate the cause. There is also a second solar pointing check that occurs 15 minutes into the orbit. This threshold is $10^{\circ}$ since SORCE is assumed to be solar pointed at this point in the orbit.

The next telemetry check involves the battery state of health. As the electronics degrade with age and repeated power cycling, there are rare orbits with unusually high battery currents. This check informs the FC if anomalous changes in the battery are detected.

Currently, the majority of brownout orbits consist of only the APE browning out. In rare circumstances when the batteries are not performing well, the UL Card can brownout in addition to the APE. Because the latter results in all spacecraft clocks resetting to the epoch time of January 1, 1980, the spacecraft time is jammed to ground time at the beginning of every orbit when the OBC is turned on, regardless of if the UL Card browned out. Another telemetry check monitors the APE FSW version to determine if the APE browned out, booted to the default FSW version, and successfully rebooted to the updated FSW.

While the original FDC is significantly altered, it still sends SORCE to safemode if an unexpected problem occurs. 
Under normal circumstances, safemode entry is cause for alarm and immediate response from the FOT. However, in DO-OP mode, safemode is a desired configuration. While the spacecraft can't conduct science, it is correctly configured for eclipse. Since the spacecraft is in a healthy state, the FOT will not take any corrective action as the spacecraft will be reconfigured the next orbit for science.

Extensive battery state of health checks are performed to monitor pressure, voltage, and temperatures. The batteries are in state of flux as occasionally good cells fail and bad cells recover. Both high and low limits are checked across these values to ensure the FOT is informed of any degradation or the recovery of the battery. These limits are updated in response to changes in the battery. The FOT is alerted by page to changes in trending beyond those that are already understood. These telemetry thresholds can be parsed by external software and compared to recent data to determine if the thresholds will be violated due to steadily increasing or decreasing trends.

The final telemetry check monitors the APE and OBC health to verify that the correct FSW is running and that each computer is responding as expected. Under normal circumstances, these checks always pass, but in the event that something unexpected happens during orbit day, these checks provide insight into the behavior of the computers.

Limit checking is disabled fifteen seconds before the end of the contact. This prevents bad data from triggering an erroneous response from the pass proc. Bad data can be introduced either due to antenna geometry or for ground contacts, atmospheric distortion as the elevation of the contact decreases relative to the ground station.

For TDRS contacts, SORCE may experience poor communications if the angle between SORCE's omnidirectional antennae and the TDRS is such that there is an antenna null. These poor quality contacts are typically avoided when scheduling TDRS contacts, but TDRS coverage requirements, SORCE's views of the TDRS spacecraft, and competition with the rest of the TDRS user community sometimes makes this impossible. Finally, at the end of the contact, connections to the ground terminal are terminated and pass proc ends. The day proc, which pauses while the pass proc runs, advances to the next contact to repeat the entire process.

\section{Command Proc}

The cmd proc, operating in conjunction with the day proc, is a unique procedure that spans the entire active schedule. However, instead of containing unique contacts, the cmd proc is uniquely configured for each orbit. The main tasks it performs are to configure the spacecraft for orbit day activities while still in safemode, load an ATS, and dump spacecraft event messages to the real time stream if in a TDRS contact. The cmd proc verifies that it is running correctly when the procedure is first started. For each orbit, the script performs the following process:

1) The cmd proc checks that autonomy for the previous orbit completed successfully. If it finds that something is still running in the specified CLP, it pages the FC. Normally, autonomy started by the cmd proc ends on its own at the end of an orbit, indicating a successful orbit. However, various anomalies such as failed ground station contacts at the end of an orbit can spoof the autonomy in such a way that it errors and hangs. The FC determines if the data is the problem or if SORCE experienced an anomaly. In either instance, the cmd proc will clean up and restart autonomy for the next orbit.

2) Before eclipse exit, the cmd proc starts Auto SORCE Orbit (ASO). ASO will configure the spacecraft if the APE browns out by turning on the OBC and jams the spacecraft time to the ground time while in safemode and prepares SORCE to transition to science mode.

3) Once configured, the cmd proc loads a unique ATS containing commands to transition SORCE to science mode and perform various science experiments. While rare, there are circumstances that prevent this from happening such as insufficient TDRS coverage. If the ATS load is not successful, a page is sent to the FC. The page is also sent if a successful ATS start cannot be confirmed, as in the case of an orbit with poor coverage in the latter half of orbit day.

4) After the ATS is loaded, The Fixx starts. This procedure runs for the remaining duration of the orbit and monitors the success of the ATS. In some circumstances, The Fixx can be used to clean up and fix various things that were not included in the updated FSW. Additionally, it periodically dumps spacecraft event messages to the real time stream for TDRS contacts.

Assuming the orbit is nominal and autonomy successfully completed, the cmd proc configures autonomy for the next orbit after SORCE enters eclipse.

\section{Auto SORCE Orbit}

ASO is responsible for monitoring and correcting the state of the spacecraft upon eclipse exit. This procedure runs in parallel to the pass proc, but only runs once at the start of the orbit. At sunrise, the procedure clears all stale 
data from relevant telemetry points to ensure that the telemetry checks are monitoring the most recent data. This is critical since stale telemetry can indicate that the OBC is turned on when it may be turned off if SORCE is browning out. If ASO were spoofed by bad telemetry, it would prevent the spacecraft from rebooting to the updated FSW. The spacecraft would enter eclipse with all loads turned on. This could have severe consequences and several redundancies are incorporated into automation to prevent this.

The procedure waits until 1 minute after sunrise before sending commands. This 1 minute gap allows the spacecraft to turn everything on without interference from the MOC and the uplink sweep from the TDRS to complete. After waiting, ASO turns on the OBC if necessary, configures the UL Card in the event of an UL Card brownout, and jams the spacecraft time to the ground time. This process repeats three more times in the event that the previous attempt is unsuccessful. If all four iterations are unsuccessful, ASO resets the APE and the UL Card in an attempt to resolve the communication problem before turning the $\mathrm{OBC}$ on again. If unsuccessful, the script repeats the $\mathrm{OBC}$ turn on sequence and APE/UL Card reset sequence until the OBC is turned on. Once on, the state of health of the OBC is checked against expected values.

If a brownout orbit is detected, a special charge sequence is loaded to the spacecraft that accounts for the incorrect charge sequence implemented by the default FSW upon boot. On non-brownout orbits, different charge sequences may be loaded to increase or decrease battery temperatures.

Once the spacecraft is correctly configured and any expected problems have been corrected, ASO determines how long and when the spacecraft booted after exiting eclipse. This time can vary based off of the solar off point at eclipse exit. If SORCE detected sunrise late into the orbit, the boot sequence happens later than desired and ground autonomy must account for this. It can also be used to determine if SORCE experienced a backflip at eclipse exit. This requires loading an increased charge rate sequence to the spacecraft since SORCE is not pointed at the sun in the beginning of the orbit.

Finally, ASO records the watermarked telemetry points. The watermarked telemetry points are single value points that record the minimum and maximum APE voltages experienced in eclipse. They are the only data that survives eclipse. It is important that the FOT track these minima and maxima as they provide insight into battery health when discharging. They are also a good indicator of degradation over time and are used to predict when brownout occurrences might happen. After recording these values, the watermarks are cleared so they accurately record the battery performance during the next eclipse. After completing these telemetry checks, ASO finishes and the cmd proc starts the ATS load procedure.

\section{E. ATS Load Proc}

The load proc ensures that the ATS for that orbit is loaded correctly. Because no contact start and stop times are in the cmd proc, logic is built in at short intervals to determine if the spacecraft is in contact with the MOC. If not in a contact, the load proc waits before continuing the ATS load. This increases the chance for a successful ATS load. If the ATS load is unsuccessful, the load proc attempts to load the ATS again until successful. Once successful, the load proc starts an RTS that starts the ATS and ends the procedure.

\section{F. The Fixx}

The fixx, named after the British rock band, monitors SORCE for the remainder of the orbit. The script first checks if the ATS successfully transitioned SORCE to normal mode. If this check passes, the procedure skips the remaining state of health checks. If the ATS is not loaded, or the ATS is loaded but the spacecraft time is not jammed or jammed incorrectly, SORCE is commanded to safemode later in the orbit. This is important for thermal considerations. If the ATS is not loaded correctly early in the orbit, the fixx sends the spacecraft to safemode, which turns off the instruments and allows them to cool down to undesirable temperatures. To prevent this, a timer on board the spacecraft is monitored to determine when the spacecraft should be sent to safemode. This timer begins when SORCE successfully exits safemode. Once the timer hits 40 minutes or it is 3 minutes before eclipse entrance, the fixx starts the safemode sequence to configure SORCE for eclipse. While it is preferred that the ATS send SORCE to safemode, this method acts as a redundant check. If no ATS is loaded and there is no contact at the end of orbit day, the spacecraft detects the solar array current dropping below a threshold and executes the safemode sequence.

The next telemetry check monitors the capacity of the battery. During brownout orbits, the default FSW can cause SORCE to point off the sun due a failed reaction wheel the FSW doesn't account for. This off point causes the solar array current to dip below the threshold and run the safemode sequence since SORCE thinks it is entering eclipse. Once the spacecraft recovers to nominal sun pointing, it is likely that the battery is not properly charged by the default version 
of FSW. If ASO was unsuccessful in loading the correct charge sequence or if the commands in the ATS to start the charge sequence have expired, the fixx will load and start the correct charge sequence.

Once all checks are performed, the fixx dumps the spacecraft event messages to the real time stream every 5 minutes during all TDRS contacts. The FSW generates event messages to inform and record what the spacecraft is doing in a non-numerical way. Logic is built into the script to prevent the spacecraft from dumping these messages when there is no TDRS contact. At the end of the orbit, the fixx notifies the FC by email if the spacecraft did not achieve normal mode. If normal mode is not achieved for 3 or more consecutive orbits the FC is paged.

\section{Conclusion}

Since 2014, SORCE is successfully operating in DO-OP mode and regularly exceeds all science requirements. With minimal adjustments, ground automation has successfully configured the spacecraft for over 2,000+ orbits and 60,000+ contacts. During this time, ground autonomy has correctly responded to several anomalies such as APE brownout, UL Card brownout, battery degradation and recovery, unexpectedly high battery currents, various connection issues with ground stations, and TDRS anomalies. Without ground automation, DO-OP mode would require an unsustainable commitment from the FOT. By eliminating constant manual commanding and creating procedures that can completely configure the spacecraft by reacting to a variety of nominal and anomalous conditions, the FOT has developed a low risk solution to allow SORCE to continue science operations in a demanding environment. As components degrade, the FOT updates and adds additional capabilities to the ground automation to keep SORCE healthy and performing nominally. 